\title{
Albedo à superfície a partir de imagens Landsat 5 em áreas de cana-de-açúcar e cerrado ${ }^{1}$
}

\author{
Pedro R. G iongo ${ }^{2}$, Geber B. de A. Moura ${ }^{3}$, Bernardo B. Silva ${ }^{4}$, Humberto R. da Rocha ${ }^{5}$, Sérgio R. R. de Medeiros ${ }^{2} \&$ Aérica C. Nazareno ${ }^{2}$
}

\section{RESU MO}

Propôs-se, neste trabalho, estimar dados de albedo à superfície terrestre usando-se o sensor Thematic Mapper (TM) do satélite LANDSAT 5 e compará-lo com dados de duas estações agrometeorológicas localizadas em região de Cerrado e a outra em cultivo da cana-de-açúcar. A região de estudo está localizada no município de Santa Rita do Passa Q uatro, SP, Brasil. Para a realização do estudo obtiveram-se seis imagens orbitais do satélite Landsat 5 sensores TM, na órbita 220 e ponto 75 , nas datas de 22/02,11/04,29/05, 01/08, 17/08 e 21/11, todas do ano de 2005, a que correspondem os dias juliano de 53, 101, 149, 213, 229 e 325, respectivamente. As correções geométricas para as imagens foram realizadas e geradas as cartas de albedo. 0 algoritmo SEBAL estimou satisfatoriamente os valores de albedo de superfícies sobre áreas de cerrado e de cana-de-açúcar, na região de Santa Rita do Passa Q uatro, SP, consistentes com observações realizadas do albedo à superfície.

Palavras-chave: sensoriamento remoto, radiação atmosférica, SEBAL

\section{Surface albedo from Landsat 5 images in areas of sugar cane and cerrado}

\begin{abstract}
This study aimed to estimate albedo data from the land surface sensor using the images of Thematic Mapper (TM) satellite LANDSAT 5 and to compare it with data from two agrometeorological stations located in the region of Cerrado, and another in sugar cane cultivation. The study area is located in the municipality of Santa Rita do Passa Q uatro - SP, Brazil. To carry out the study six orbital images were obtained from the satellite Landsat 5 TM sensors in the orbit 220 and in the section 75, for the dates of 22/02,11/04, 29/05, 01/08, 17/08 and 21/11 (all in the year of 2005) which correspond to the days $53,101,149,213,229$ and 325 , respectively. The geometric correction for images was performed and the letters of albedo were generated. The algorithm SEBAL estimated, satisfactorily, the values of albedo on the surface areas of Cerrado and sugar cane in the region of Santa Rita do Passa Quatro - SP, consistent with observations made of the surface albedo.
\end{abstract}

Key words: sensing remote, atmospheric radiation, SEBAL

\footnotetext{
1 Parte da Dissertação de Mestrado do primeiro autor apresentada a UFRPE

2 Mestre em Engenharia Agrícola U FRPE, Av. Dom Manoel de Medeiro s/n, Dois Irmãos, CEP 52171-900, Recife, PE. Fone: (81) 3320-6246. E-mail: giongopr@yahoo.com.br; medeirossoft@gmail.com; aericacn@yahoo.com.br

3 DEPA/UFRPE. Fone: (81) 3320-6246. E-mail: geber@depa.ufrpe.br

4 DCA/UFCG, Av. Aprígio Veloso, 822, Bodocongó, CEP 58109-080, Campina Grande, PB. Fone: (81) 3310-1054. E-mail: bernardo@dca.ufcg.edu.br

5 IAG/U SP. Rua do Matão, 1226, Cidade Universitária, CEP 05508-090, São Paulo, SP. Fone: (11) 3091-4762. E-mail: humberto@model.iag.usp.br
} 


\section{INTRODUÇÃO}

A principal vantagem do sensoriamento remoto é a geração de informações com rapidez e a custo moderado; nas aplicações como saldo de radiação, faz-se necessário o conhecimento do albedo visto que, se existisse uma malha de estações de superfície, seria difícil gerenciá-las e mantê-las em funcionamento pelo custo de manutenção das mesmas e outros agravantes; assim, imagens de satélite têm cobertura, atualmente, de praticamente todo o planeta e com alta resolução para esses determinados estudos.

Segundo Silva et al. (2005a) o saldo de radiação é de fundamental importância nos processos de troca de calor e massa na baixa troposfera, uma vez que se constitui no principal responsável pelo aquecimento do solo, do ar e, sobretudo, pela evapotranspiração da vegetação nativa e das culturas.

Bastiaanssen (1995) desenvolveu o Surface Energy Balance algorithm for Land (SEBAL) validando-o com campanhas experimentais na Espanha e no Egito, utilizando imagens do satélite Landsat 5-TM; logo adiante, Roerink et al. (1997) também aplicaram o mesmo sensor para monitorar o desempenho de irrigação na Argentina e no Paquistão, em conjunto com o sensor AVHRR/NOAA. Combinações do satélite Landsat 5-TM e NOAA/AVHRR foram usadas também por Timmermans \& Meijerink (1999) na África.

Hafeez et al. (2002) aplicaram o SEBAL através do sensor ASTER, nas Filipinas, concluindo que a combinação de alta-resolução espacial dos sensores ETM+ e ASTER com a alta resolução temporal dos sensores AVHRR e MODIS forneceu alta precisão nos estudos de balanços hídricos e usos da água, em escalas regionais.

Segundo Trezza (2002), o SEBAL é um modelo de processamento de imagens de satélite com grandes potencialidades. Allen et al. (2002) citam que este modelo se baseia no balanço superficial de energia, no qual é necessário o conhecimento do albedo em escala regional e pode ser utilizado com imagens digitais coletadas por qualquer satélite orbital que registre a radiação nos comprimentos de onda do visível, infravermelho próximo e infravermelho termal.

O SEBAL tem sido utilizado para estimar, de forma simples e eficaz, o albedo e a temperatura da superfície com imagens Landsat 5 (Bastiaanssen et al., 1998; Boegh et al., 2002; Silva et al., 2005a) e AVHRR-NOAA (Liang, 2002).

Objetivou-se, com este trabalho, estimar o albedo de superfície através de dados do sensor Thematic Mapper (TM) do satélite LANDSAT 5 e comparação de resultados estimados por satélite com dados instrumentais à superfície, em duas estações agrometeorológicas localizadas na área de estudo.

\section{MATERIAL E MÉTODOS}

A área de estudo do presente trabalho compreende uma região do município de Santa Rita do Passa Quatro, Estado de São Paulo.

Segundo Batalha (1997) a caracterização da área descrita como cerrado é Cerrado "sensu scricto”, composto predo- minantemente de arbustos e arvoretas com cerca de $5 \mathrm{~m}$ de altura, em grande adensamento, dificultando a locomoção e restringindo o campo de visão. Árvores emergentes, com cerca de 7 a $10 \mathrm{~m}$ de altura, surgem eventualmente. O componente herbáceo-subarbustivo é contínuo, mais desenvolvido que no cerradão; é a fisionomia mais frequente na reserva ocupando 79,0\% de sua área total, que é cerca de 1060 ha.

Obtiveram-se, para a realização do estudo, imagens em dias de céu claro, na órbita 220 ponto 75, correspondentes a seis dias, que são: 22-02, 11-04, 29-05, 16-07, 17-08 e 21-11 todos do ano de 2005, os quais se referem ao dia Juliano (DJ) 53, 101, 149, 213, 229 e 325, respectivamente. As imagens do Landsat 5 TM foram adquiridas junto ao Instituto Nacional de Pesquisas Espaciais (INPE), órgão ligado ao Ministério da Ciência e Tecnologia.

As imagens Landsat 5 TM são compostas de sete bandas espectrais cujas características estão presentes na Tabela 1, na qual também estão incluídas a irradiância solar monocromática $\left(\mathrm{k}_{\lambda_{\mathrm{i}}}\right)$ das bandas reflectivas (bandas 1,2 , 3, 4, 5 e 7), incidente sobre uma superfície normal à direção dos raios solares no topo da atmosfera terrestre, e a distância de uma Unidade Astronômica (UA) do Sol.

Tabela 1. D escrição das bandas do Mapeador Temático (TM) do Landsat 5 com os correspondentes intervalos de comprimento de onda, coeficientes de calibração (radiância mínima - a e máxima - b) e irradiâncias espectrais no topo da atmosfera $\left(T_{O A}\right)$

\begin{tabular}{|c|c|c|c|c|}
\hline \multirow{2}{*}{ Bandas } & \multirow{2}{*}{$\begin{array}{l}\text { Comprimento } \\
\text { de Onda } \\
(\mu \mathrm{m})\end{array}$} & \multicolumn{2}{|c|}{$\begin{array}{l}\text { Coeficientes de Calibração } \\
\left(\mathrm{W} \mathrm{m} \mathrm{m}^{-2} \mathrm{sr}^{-1} \mathrm{~nm}^{-1}\right)\end{array}$} & \multirow{2}{*}{$\begin{array}{c}\mathrm{T}_{\mathrm{OA}} \\
\left(\mathrm{W} \mathrm{m} \mathrm{m}^{-2} \mathrm{~m}^{-1}\right)\end{array}$} \\
\hline & & a & b & \\
\hline 1 (azul) & $0,45-0,52$ & $-1,52$ & 193,0 & 1967 \\
\hline 2 (verde) & $0,52-0,60$ & $-2,84$ & 365,0 & 1826 \\
\hline 3 (vermelho) & $0,63-0,69$ & $-1,17$ & 264,0 & 1554 \\
\hline 4 (IV-próximo) & $0,76-0,79$ & $-1,51$ & 221,0 & 1036 \\
\hline 5 (IV-médio) & $1,55-1,75$ & $-0,37$ & 30,2 & 215,0 \\
\hline 6 (IV-termal) & $10,4-12,5$ & 1,2378 & 15,303 & - \\
\hline 7 (IV-médio) & $2,08-2,35$ & $-0,15$ & 16,5 & 80,67 \\
\hline
\end{tabular}

Em estudos de áreas de inclinação considerável, o ângulo de incidência da radiação solar depende da inclinação da superfície e do seu aspecto, isto é, do azimute normal da superfície; assim, para a área de estudo se obteve o modelo de elevação digital do terreno (DEM), gratuitamente, no site do SRTM (2007) referente ao mosaico x 27 e y 17, após se realizar o download do arquivo referente ao local de estudo; foram reamostrados, então, os pixeis, para $30 \times 30 \mathrm{~m}$, visto que os dados do DEM são disponíveis aos pixeis com uma resolução espacial de 90 x $90 \mathrm{~m}$.

O sensor TM mede a radiância espectral dos alvos e os armazena na forma de níveis de cinza ou intensidade do pixel ou, ainda, número digital (ND), cujos valores variam de 0 a 255 (8 bits), com uma resolução espacial de $30 \mathrm{~m}$ nas bandas 1, 2, 3, 4, 5 e 7, e outra de $120 \mathrm{~m}$, na banda 6; desta forma, para a visualização e realização de operações matemáticas intra e interbandas, recortes, classificação e outras operações, utilizou-se o software ERDAS Imagine 8.7. 
Para o processamento das imagens são desenvolvidos modelos matemáticos através da ferramenta Model Maker do ERDAS Imagine 8.7.

\section{Radiância espectral}

Para o cômputo da radiância espectral de cada banda $\left(\mathrm{L}_{\lambda_{\mathrm{i}}}\right)$, ou seja, a efetivação da calibração radiométrica é realizada em que o número digital (ND) de cada pixel da imagem é convertido em radiância espectral monocromática; essas radiâncias representam a energia solar refletida por cada pixel, por unidade de área, de tempo, de ângulo sólido e de comprimento de onda, medida a nível do satélite Landsat (altitude de $705 \mathrm{~km}$ ), para as bandas 1, 2, 3, 4, 5 e 7, enquanto a banda 6 para referida radiância representa a energia emitida por cada pixel, segundo a equação proposta por Markham \& Baker (1987).

$$
\mathrm{L}_{\lambda \mathrm{i}}=\mathrm{a}_{\mathrm{i}}+\frac{\mathrm{b}_{\mathrm{i}}-\mathrm{a}_{\mathrm{i}}}{255} \mathrm{ND}
$$

em que a e b são as radiâncias espectrais mínimas e máximas (W m ${ }^{-2} \mu \mathrm{m}^{-1}$, Tabela 1); ND é a intensidade do pixel (número digital - número inteiro de 0 a 255) e i corresponde às bandas $(1,2,3, \ldots$ e 7$)$ do satélite Landsat 5 - TM.

\section{Refletividade}

O cômputo da reflectância monocromática de cada banda $\left(\rho_{\lambda}\right)$ é definido como sendo a razão entre o fluxo de radiação refletida e o fluxo de radiação incidente que é obtida segundo a equação Allen et al. (2002).

$$
\rho_{\lambda \mathrm{i}}=\frac{\pi \cdot \mathrm{L}_{\lambda \mathrm{i}}}{\mathrm{k}_{\lambda \mathrm{i}} \cdot \cos Z \cdot \mathrm{dr}}
$$

donde $\mathrm{L}_{\lambda_{\mathrm{i}}}$ é a radiância espectral de cada banda, $\mathrm{k}_{\lambda_{\mathrm{i}}}$ é a irradiância solar espectral de cada banda no topo da atmosfera ( $\mathrm{W} \mathrm{m}^{-2} \mu \mathrm{m}^{-1}$ ), Z é o ângulo zenital solar e dr é o inverso do quadrado da distância relativa Terra-Sol (em unidade astronômica - UA), dada por Iqbal, (1983).

$$
\mathrm{dr}=1+0,033 \cos \left(\frac{\mathrm{DJ} 2 \pi}{365}\right)
$$

O ângulo zenital foi obtido com a seguinte fórmula.

$$
\operatorname{Cos} Z=\cos \left(\frac{\pi}{2}-E\right)
$$

donde: E é o ângulo de elevação do sol, obtido no cabeçalho de cada imagem.

\section{Albedo planetário}

$O$ albedo planetário $\left(\alpha_{\text {toa }}\right)$ é aquele não ajustado à transmissividade atmosférica a qual, por sua vez, é obtida pela combinação linear das reflectâncias monocromáticas segundo a Eq. 5.

$\alpha_{\text {toa }}=0,293 \rho_{1}+0,274 \rho_{2}+0,233 \rho_{3}+0,157 \rho_{4}+0,033 \rho_{5}+0,011 \rho_{7}$ em que: $\rho_{1}, \rho_{2}, \rho_{3}, \rho_{4}, \rho_{5}$ e $\rho_{7}$ são as reflectância das bandas $1,2,3,4,5$ e 7 .

\section{Albedo da superfície}

Após a obtenção do albedo planetário fez-se o cômputo do albedo da superfície ou albedo corrigido para os efeitos atmosféricos $\alpha$, pela Eq. 6.

$$
\alpha=\frac{\alpha_{\mathrm{toa}}-\alpha_{\mathrm{p}}}{\tau_{\mathrm{sw}}{ }^{2}}
$$

donde $\alpha_{\text {toa }}$ é o albedo planetário, $\alpha_{\mathrm{p}}$ é a radiação solar refletida pela atmosfera, que varia entre 0,025 e 0,04, mas para o modelo SEBAL é recomendado o uso do valor de 0,03, com base em Bastiaanssen (2000) e $\tau_{\mathrm{sw}}$ é a transmissividade atmosférica que, para condições de céu claro, proposta por Allen et al. (2007) pode ser escrita como a Eq. 7.

$$
\mathrm{t}_{\mathrm{sw}}=0,35+0,627 * \mathrm{EXP}\left[\frac{-0,00146 \mathrm{P}}{\mathrm{k}_{\mathrm{t}} \cos \mathrm{Z}}-0,075\left(\frac{\mathrm{W}}{\cos \mathrm{Z}}\right)^{0,4}\right]
$$

sendo: $\mathrm{P}$ a pressão atmosférica $(\mathrm{kPa})$ obtida pela Eq. $8, \mathrm{k}_{\mathrm{t}}$ um coeficiente de turbidez da atmosfera que tem valores de $0<\mathrm{k}_{\mathrm{t}} \leq 1$ com base em Allen (1996), os valores atribuídos para cada DJ são apresentados na Tabela 2, cosZ já foi introduzido anteriormente e $\mathrm{W}$ é função da quantidade de água precipitável em um ponto da imagem (local da estação agrometeorológica) e foi calculada pela Eq. 9, proposta por Garrison \& Adler (1990).

Tabela 2. Valores de coeficiente de turbidez $\left(k_{t}\right)$, atribuídos para cada DJ, referente às imagens para Santa Rita do Passa Q uatro, SP, 2005

\begin{tabular}{ccccccc} 
DJ & $\mathbf{5 3}$ & $\mathbf{1 0 1}$ & $\mathbf{1 4 9}$ & $\mathbf{2 1 3}$ & $\mathbf{2 2 9}$ & $\mathbf{3 2 5}$ \\
$\mathrm{k}_{\mathrm{t}}$ & $\mathbf{1 , 0}$ & 1,0 & 0,6 & 0,4 & 0,4 & 0,6 \\
\hline
\end{tabular}

Os valores de $k_{t}$, foram atribuídos para esses dias, com base nos valores de concentração de $\mathrm{CO}_{2}$ (ppm) obtidos na estação agrometeorológica, localizada na área de estudo; observaram-se valores menores de $\mathrm{CO}_{2}$, iguais a: 382, 370, 401, 397, 399 e 398 ppm, para os DJ 53, 101, 149, 213, 229 e 325; com relação aos valores para os DJ 213 e 229, não houve aumento considerável na concentração de $\mathrm{CO}_{2}$ porém se sabe que esses dias coincidem com o período de queimadas e colheita da cultura da cana o que torna os valores de turbidez menores (Tabela 2).

$$
\mathrm{P}=101,3\left(\frac{\mathrm{T}_{\mathrm{ar}}-0,0065^{*} \mathrm{~h}}{\mathrm{~T}_{\mathrm{ar}}}\right)^{5,26}
$$

em que: $\mathrm{T}_{\mathrm{ar}}$ é a temperatura do ar (K) que na Tabela 3 se encontra em ${ }^{\circ} \mathrm{C}$ e h é a altitude média da superfície em ralação ao nível médio do mar (m) que, para este estudo, foi considerado média da região em $600 \mathrm{~m}$.

$$
\mathrm{W}=0,14 * \mathrm{e}_{\mathrm{a}} \mathrm{P}_{\text {air }}+2,1
$$

sendo: $e_{a}$ a pressão de vapor de água $(\mathrm{kPa})$, valores que 
estão na Tabela 3, $\mathrm{P}_{\mathrm{ar}}$ os valores da pressão atmosférica obtidos na estação agrometeorológica na área de estudo e também estão na Tabela 3, para cada DJ referente aos dias das imagens e W é a quantidade de água precipitável encontrada na atmosfera, em mm.

Obtiveram-se, para comparação dos dados gerados com imagens do sensor TM, os dados de superfície de duas estações agrometeorológicas localizadas na área de estudo, ressaltando-se que uma das torres se encontrava em área cultivada com cana-de-açúcar (USR), e a segunda estação estava localizada em área de cerrado (PDG).

Tabela 3. Dados da estação agrometeorológica correspondentes ao dia juliano (DJ) e horário da passagem do satélite na área estudada

\begin{tabular}{rccc}
\hline DJ & $\mathbf{e}_{\mathbf{a}}(\mathbf{m b})$ & $\mathbf{P}_{\text {air }}(\mathbf{k P a})$ & $\mathbf{T}_{\text {ar }}\left({ }^{\circ} \mathbf{C}\right)$ \\
53 & 1,9948 & 948 & 26,14 \\
101 & 2,1169 & 954 & 26,63 \\
149 & 1,5971 & 950 & 20,23 \\
213 & 1,3688 & 950 & 21,59 \\
229 & 1,6588 & 946 & 23,72 \\
325 & 2,0218 & 950 & 24,52 \\
\hline
\end{tabular}

$e_{a}$ - pressão de vapor da água; $P_{\text {air }}$ - pressão atmosférica; $T_{a r}$ - temperatura do ar

\section{RESULTADOS E DISCUSSÃO}

Na Figura 1 se encontram o recorte de uma imagem da área de estudo e a localização das estações agrometeorológicas da área de cana-de-açúcar e do cerrado, com as coordenadas geográficas da estação da cana-de-açúcar com latitude de $21^{\circ} 38^{\prime} 13^{\prime}$ ' S e longitude de $47^{\circ} 47^{\prime} 25^{\prime}$ ' W, a estação do cerrado $21^{\circ} 37^{\prime}$ ' ' S e $47^{\circ} 37^{\prime}$ '56” W e, ainda, o lago com $21^{\circ} 36^{\prime} 22^{\prime}$ ' S e $47^{\circ} 47^{\prime} 54^{\prime}$ ' W; a altitude média da região é de $600 \mathrm{~m}$.
O recorte da imagem resultou em um retângulo, com as seguintes coordenadas do canto superior esquerdo, de $21^{\circ} 34^{\prime} 45^{\prime \prime} \mathrm{S}$ e $47^{\circ} 49^{\prime} 13^{\prime \prime} \mathrm{W}$, e no canto inferior direito com $21^{\circ} 40^{\prime} 52^{\prime}$ ' S e $47^{\circ} 36^{\prime}$ '23” W, correspondendo a 746 x 364 pixeis, além de uma área de aproximadamente 24.439 ha.

Os valores de transmissividade calculados para cada dia foram de $0,736,0,721,0,659,0,631,0,636$ e 0,723 para os dias 53, 101, 149, 213, 229 e 325, respectivos aos dias das imagens.

Apresentam-se, na Tabela 4, os valores estatísticos das cartas de albedo da superfície gerados pelo algoritmo proposto para os dias de estudo.

Tabela 4. Valores dos parâmetros estatísticos mínimo, máximo, médio, mediano, moda e desvio padrão (D.PAD), obtidos na análise estatística das cartas de albedo da superfície

\begin{tabular}{rrrrcrc}
\hline DJ & mínimo & máximo & médio & mediano & moda & D.PAD \\
53 & 0,019 & 0,332 & 0,135 & 0,129 & 0,104 & 0,030 \\
101 & 0,025 & 0,291 & 0,133 & 0,129 & 0,103 & 0,027 \\
149 & 0,032 & 0,377 & 0,156 & 0,151 & 0,118 & 0,037 \\
213 & 0,028 & 0,446 & 0,152 & 0,145 & 0,121 & 0,036 \\
229 & 0,028 & 0,447 & 0,156 & 0,148 & 0,128 & 0,034 \\
325 & 0,013 & 0,371 & 0,126 & 0,114 & 0,110 & 0,039 \\
\hline DJ - dia juliano & & & & &
\end{tabular}

Adotaram-se, para as cartas de albedo de superfície, os valores menores mínimos nas imagens dos DJ 53 e 325, com valores de 1,9 e 1,3\%, respectivamente, inferiores, portanto, aos valores obtidos por Silva et al. (2005a) que foram de 6,8 e 7,4\% para os anos de 2000 e 2001, respectivamente. Mendonça (2007) obteve valor 0 para albedo em estudo com imagens MODIS.

Ainda em referência aos maiores valores máximos, observaram-se, nas cartas dos dias 213 e 229, valores de 44,6 e $44,7 \%$, respectivamente, conforme a Tabela 4 , sendo que

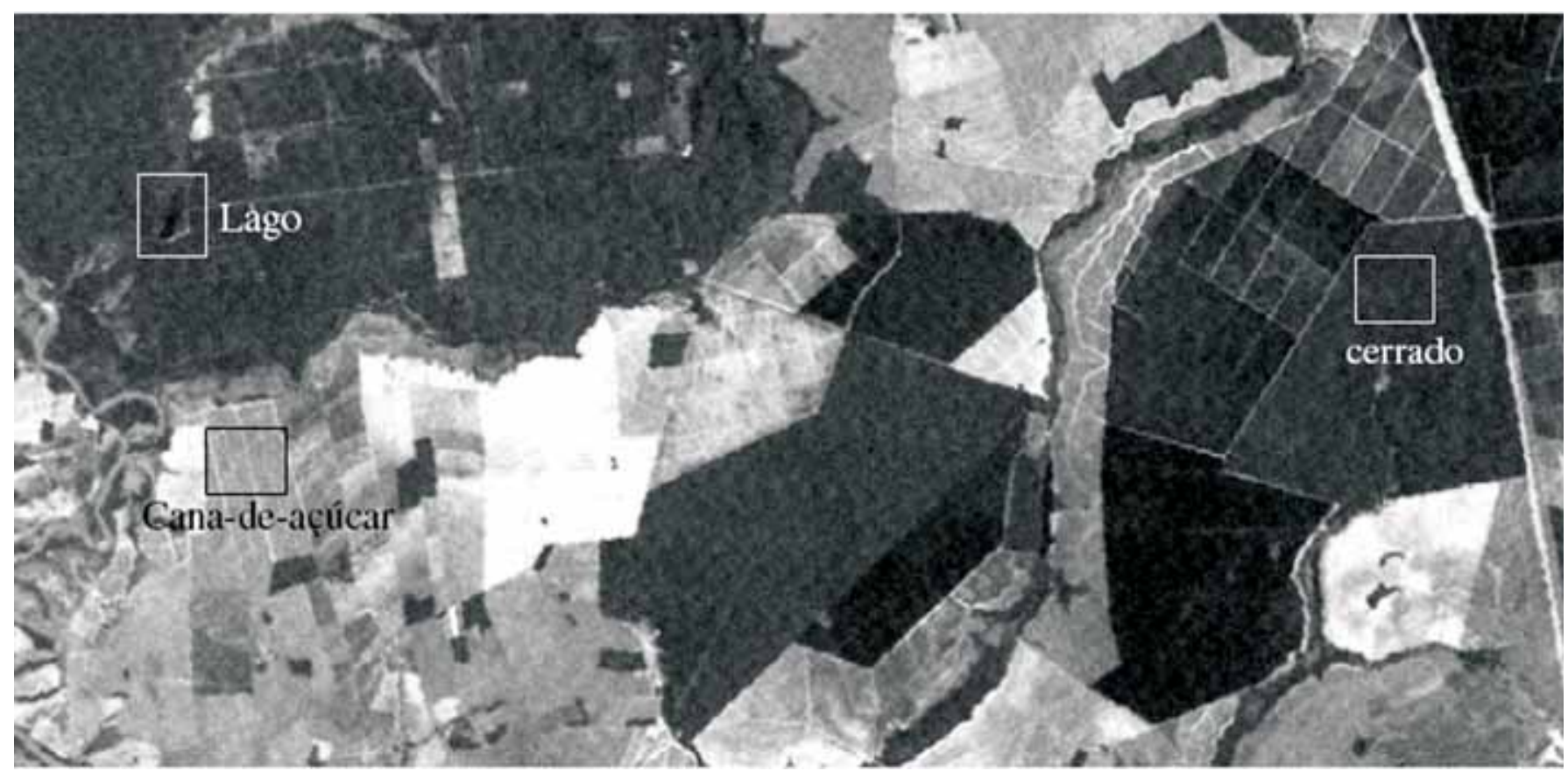

Figura 1. Imagem do DJ 53 composição das bandas R-G -B 4-3-2, do satélite LAN DSAT 5 - TM e, local ização das estações agrometeorológica na área de estudo 
esses valores se encontram superiores aos observados por Mendonça (2007), que encontrou valores máximos de 31 e $31 \%$ para albedo, sendo que a variação da composição na cobertura da superfície pode alterar significativamente os valores de mínimo e/ou máximos em uma cena estudada.

Também se constatou, para os valores médios, comportamento semelhante ao dos valores mínimos e máximos, sendo que as médias obtidas no período apresentaram, nas cartas dos DJ de 325 e 149, valores de 12,6 e 15,6\%, respectivamente. Mendonça (2007) ainda encontrou valores médios nas cartas de albedo de 13 a 18\%, muito próximos aos obtidos nesta pesquisa, enquanto Silva et al. (2005a) obtiveram médias de 20,5 e 21\% nos anos de 2000 e 2001, respectivamente, superiores aos obtidos neste trabalho porém são justificados pela diferença de alvos estudados em cada trabalho.

Observou-se, em relação ao albedo, que a dispersão dos valores em cada carta foi pequena quando se verificaram os valores de desvio padrão, apresentados na Tabela 3; supõe-se que esta pequena variação dos valores seja devido, à pouca hete- rogeneidade da vegetação dentro de cada carta obtida.

Encontram-se, na Figura 3, as cartas de albedo de superfície para os dias que se referem ao DJ das imagens que os menores valores foram obtidos em todas as cartas, em superfícies do lago e áreas adjacentes, como verificado na Figura 3A a F com coloração cinza-escuro, enquanto os maiores valores foram obtidos em áreas de cultivo e áreas expostas (sem cobertura), com coloração cinza-claro, para a Figura 3F, na sua parte superior.

Tem-se, na Figura 3F, uma classe bem destacada, com cor cinza-claro, a qual apresentou valores médios superiores a $27 \%$ de albedo, caracterizada como área de solo exposto, tal como Silva et al. (2005b) encontraram valores de albedo para solo exposto de 31 e 33\% para os anos de 2000 e 2001, respectivamente, valores muito coerentes com os obtidos nesta pesquisa.

Também Boegh et al. (2002) obtiveram valores em estudo na Dinamarca com imagens Landsat 5 TM em áreas de vegetação densa igual a 18\%. Silva et al. (2005a), em estudo com o mesmo sensor e satélite, notaram albedo semelhante
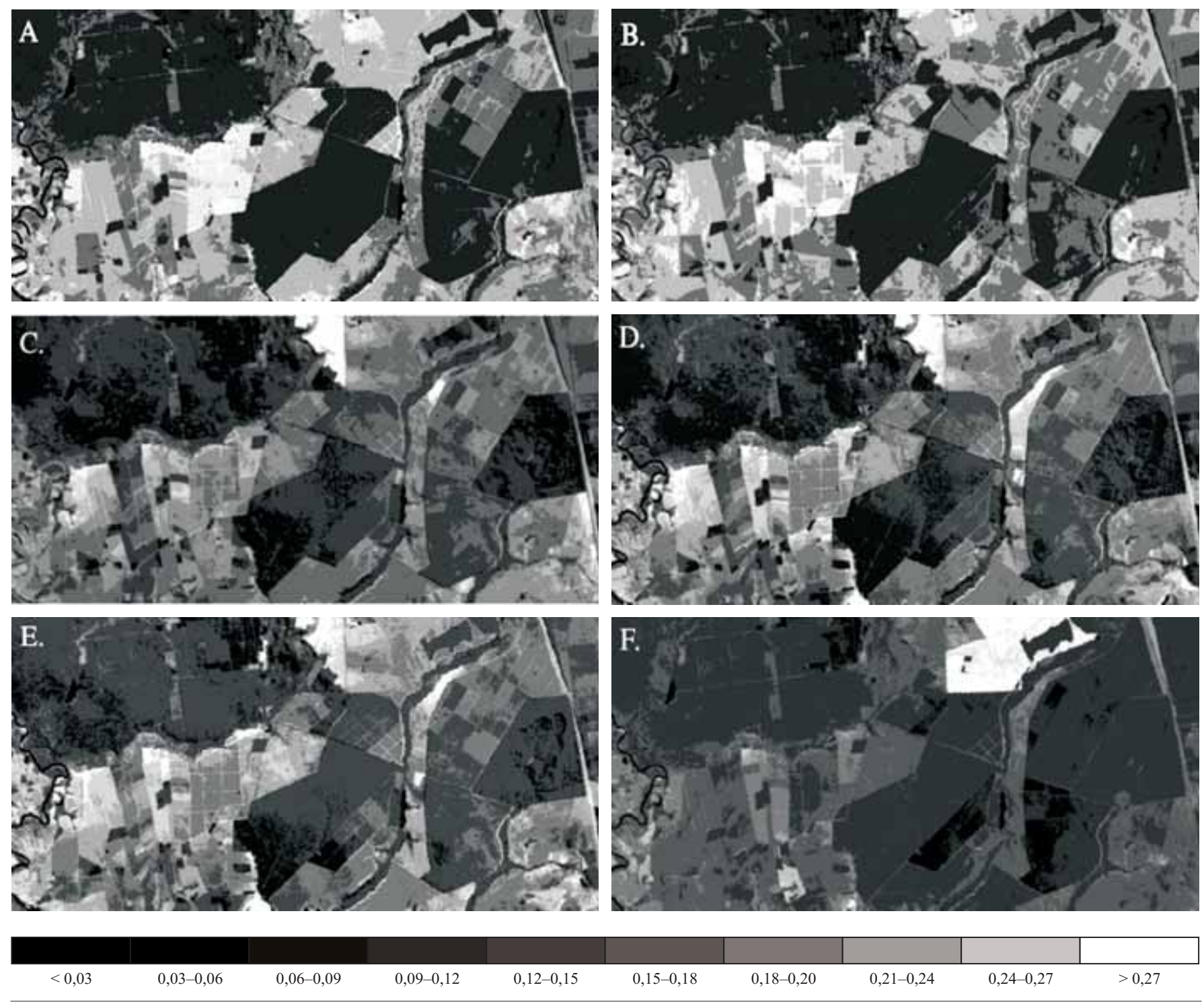

Figura 2. Carta de albedo referente aos DJ 53 (A), 101 (B), 149 (C), 213 (D), 229 (E) e 325 (F) 
aos que foram citados anteriormente, corroborando com os valores obtidos nesta pesquisa.

Iziomons \& Mayer (2002), avaliando o comportamento do albedo em áreas de pastagens no sudoeste da Alemanha, entre 1991 e 1996, constataram acentuada variabilidade no período estudado, com valores situados entre 22,2\% a 36\%.

Chandrapala \& Wimalasuriya (2003) obtiveram albedo de 34 a 36\%, em grandes extensões de dunas de areia, com grande estabilidade no período de julho de 1999 a julho de 2000.

Foi transparente a alteração no albedo de diferentes alvos quando comparados com as cartas de todo o período, sendo que a variação anual do albedo é fonte de diversos fatores, dentre eles a variação na composição da cobertura, a qual sofre variação no período, fato verificado na Figura 3.

$\mathrm{O}$ albedo médio para as cartas foi maior nas imagens 3B a $3 \mathrm{E}$, nas quais se verifica maior presença de classes de cor cinza em tons mais claros, uma vez que as datas de obtenção dessas imagens se referem ao período intermediário do ano e o sol estava no hemisfério Norte, razão pela qual o

A.

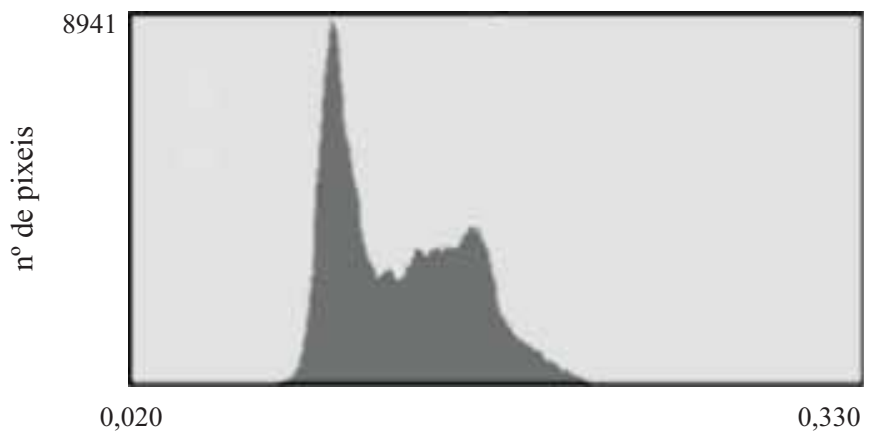

C.

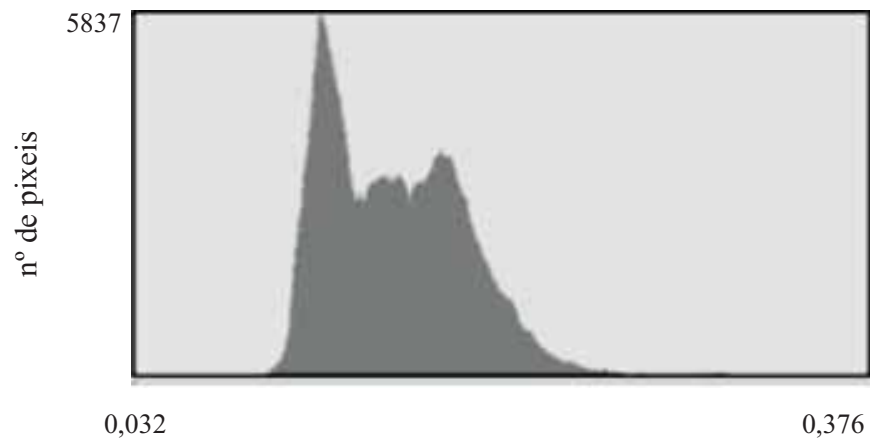

E.

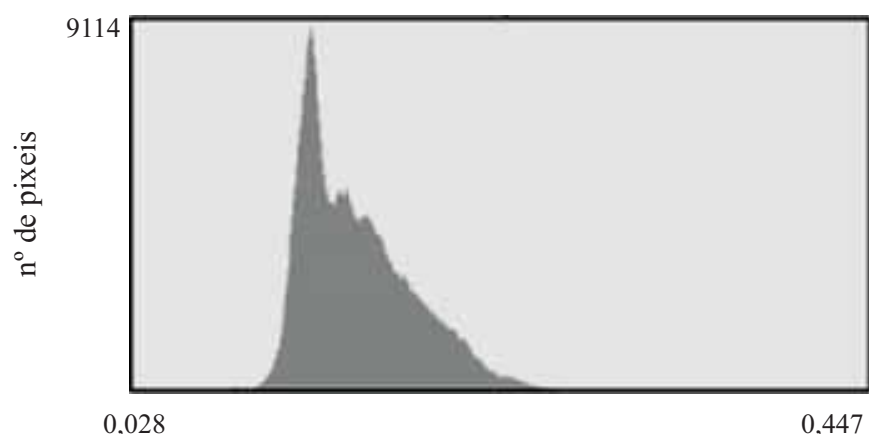

ângulo de incidência da radiação é mais facilmente refletido em relação ao ângulo de incidência da radiação para as imagens das Figuras 3A e F.

Na Figura 4 estão os histogramas de frequência dos valores de albedo para as cartas geradas neste estudo, e assim é possível verificar maior dispersão dos valores nas cartas das Figuras 4A a E, enquanto a carta 4F apresenta a maior concentração em torno da média.

Nas Figuras 4A, B e C, em que aparecem os histogramas de frequiência do albedo de todos os pixeis da área de estudo, é possível perceber duas modas bem definidas, a menor (melhor definida na Fig. 4B) se refere à superfície de vegetação de cerrado e superfícies de vegetação pouco densa e a segunda moda, de valores maiores, é de superfície com culturas como cana-de-açúcar e eucalipto, as quais possuem maior cobertura nesta área de estudo.

Buscando-se avaliar melhor o desempenho da metodologia proposta para o albedo de superfície, realizaram-se recortes de áreas homogêneas dentro da cena estudada, sendo

\section{B.}

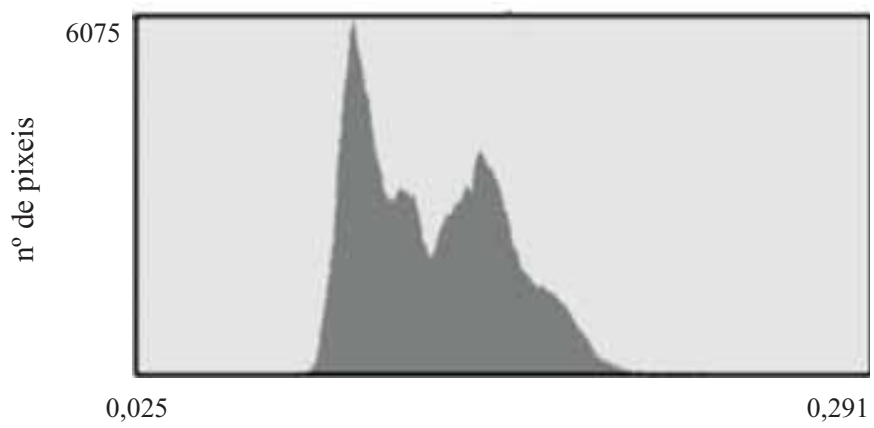

D.

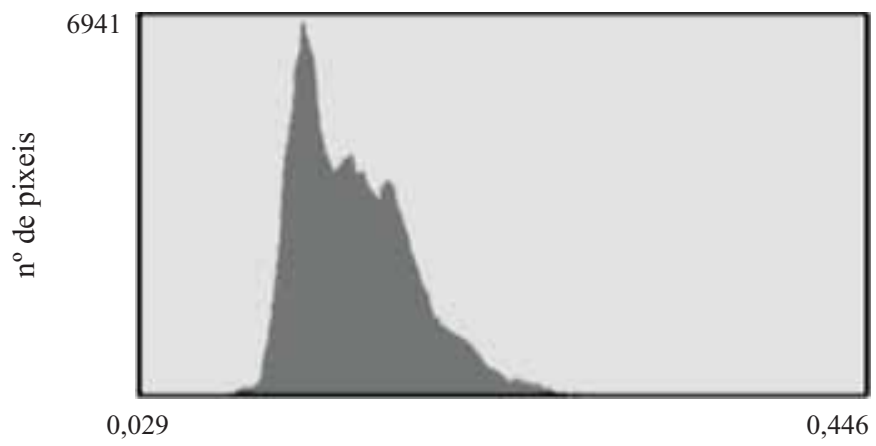

F.

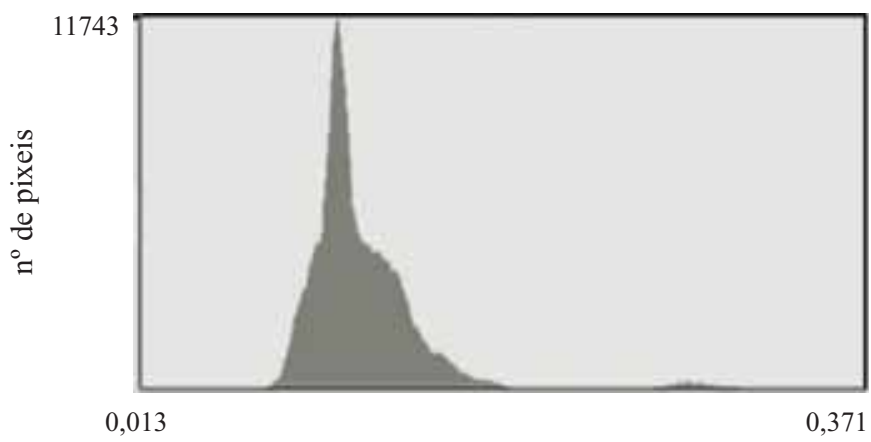

Figura 3. Histograma de frequência das cartas de al bedo para os DJ 53 (A), 101 (B), 149 (C), 213 (D), 229 (E) e 325 (F) 
então extraídos três pequenos recortes de diferentes pontos, com tamanhos de 20 a 30 pixeis, cujas áreas representam o lago (superfície com água), cana-de-açúcar e cerrado, conforme localização na Figura 2.

Os valores de albedo para água variaram entre 1,7 a $10,4 \%$ nas imagens estudadas; comportamento semelhante também foi obtido por Silva et al. (2005a) com valores entre 7 a 9\%; ainda outros autores, como Bastiaanssen et al. (1998) e Bastiaanssen (2000) encontraram valores entre 3 a $10 \%$ para albedo de superfície em superfícies com água na Bacia de Gediz, na Turquia; já Giongo et al. (2007) obtiveram valores entre 7 e 12\% para albedo em superfície de água, na região de Ibimirim, Estado de Pernambuco.

Apresentam-se, na Figura 5A, os valores dos pixeis das cartas de albedo para os dias estudados na área do lago e se verifica que os valores estão entre 2 e $8 \%$ para todas as imagens; a média se situa próximo a $4 \%$.

Os valores médios encontrados para albedo em área de cana-de-açúcar sofreram variação de 14,2 a 21,9\% e foram obtidos nos DJ, 325 e 213, respectivamente, conforme a Figura 5B.

Os valores de albedo encontrados na área de cana se situaram entre 13,5 a $23,1 \%$ para o período das imagens; es-

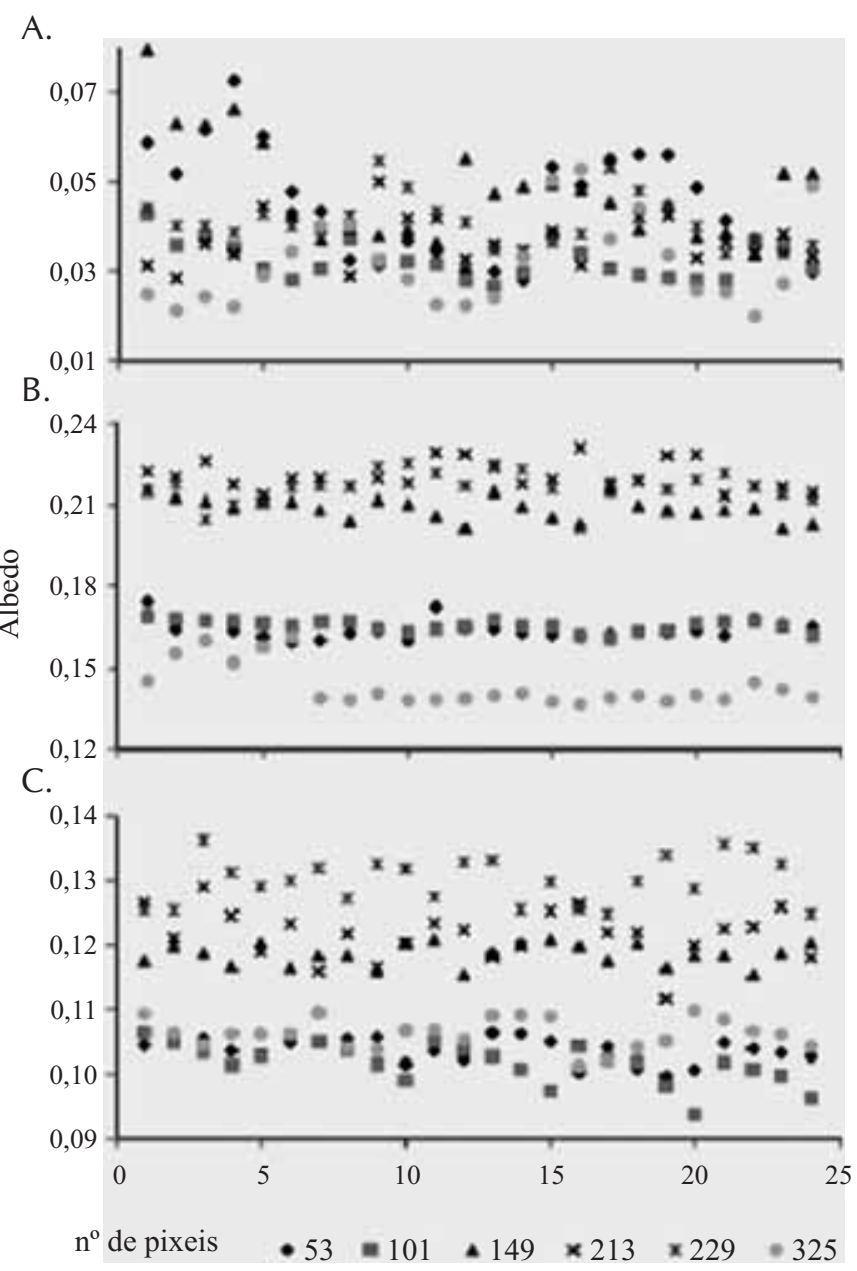

Figura 4. Valores de albedo da superfície dos pixeis do recorte na área do lago (A), cana-de-açúcar (B) e de cerrado (C) para os DJ referentes aos recortes das imagens ses valores corroboram com os obtidos por Mendonça (2007), que encontrou valores para albedo entre 14 e $22 \%$ em área de cana-de-açúcar, na região Norte Fluminense.

Varejão-Silva (2000) cita que valores de albedo para esta cultura, de $15 \%$, estão dentro, portanto, da faixa encontrada neste estudo para o período estudado.

Dois grupos de médias na Figura 5B foram observados, o menor nos dias 53, 101 e 325, com valor médio de 16\%, e as cartas são do período de primavera e verão, e o segundo foi obtido nos DJ 149, 213 e 229, com valor médio de 21\%, cujas cartas se referem aos períodos de outono e inverno.

Constatou-se, ainda, pequena variação dos valores para o recorte da cena realizada na área de cana-de-açúcar, a qual é verificada na Figura 5B.

Para o recorte das imagens de albedo de superfície na área de cerrado, Figura 5C, observaram-se os valores menores mínimos obtidos nos DJ 53 e 101, com valores de 9,6 e 9,3\%, respectivamente. Os maiores máximos foram obtidos nas cartas dos DJ 213 e 229, com valores de 13,4 e 13,6\%, respectivamente.

Os valores médios para a área de cerrado foram inferiores aos obtidos na área de cana-de-açúcar, para todo o período estudado, sendo que os médios se mantiveram entre 10,1 e $13,1 \%$, obtidos nos DJ 101 e 229, respectivamente.

Ainda segundo Varejão-Silva (2000) o valor médio para vegetação tropical é de 13\%; valores muito próximos, portanto, aos obtidos nesta pesquisa.

Querino et al. (2006) encontraram valores médios de albedo para floresta de 13\%; eles citam que existe grande variação nos valores entre o período seco e o chuvoso; tais fatores são importantes para esta variação; os autores mencionam que as mudanças de coloração por parte dos vegetais, que se tornam mais verdes no período chuvoso, e ainda solo mais escuro devido à presença da umidade, são fatores condicionantes das mudanças no albedo.

Na Figura 5C se acham os valores dos pixeis das cartas de albedo para os dias estudados na área de cerrado. O comportamento do albedo para o cerrado foi semelhante ao da área da cana, com duas médias distintas entre as imagens, sendo que a menor foi observada nos DJ 53, 101 e 325 com valores entre 9 e 11\% e para os DJ 149, 213 e 229, valores médios entre 11,5 a $13,5 \%$.

Constataram-se, para os pixeis das estações agrometeorológica do cerrado (PDG) e da cana-de-açúcar (USR), valores para o albedo da superfície, apresentados na Tabela 5; referidos valores de albedo foram estimados pela razão entre a radiação global refletida e a incidente coletada por dois

Tabela 5. Valores de al bedo obtidos nas estações agrometeorológicas do cerrado (PDG) e da cana-de-açúcar (USR) e os obtidos pelo modelo SEBAL

\begin{tabular}{rrrrrrc}
\hline DJ & $\begin{array}{c}\text { PDG } \\
\text { estação }\end{array}$ & $\begin{array}{c}\text { PDG } \\
\text { SEBAL }\end{array}$ & Erro & $\begin{array}{c}\text { USR } \\
\text { estação }\end{array}$ & $\begin{array}{c}\text { USR } \\
\text { SEBAL }\end{array}$ & Erro \\
53 & 0,1258 & 0,1029 & 0,022 & 0,1939 & 0,1663 & 0,027 \\
101 & 0,1256 & 0,0942 & 0,031 & 0,2028 & 0,1579 & 0,045 \\
149 & 0,1267 & 0,1134 & 0,013 & 0,1689 & 0,2152 & $-0,045$ \\
213 & 0,1187 & 0,1252 & $-0,006$ & 0,1871 & 0,2153 & $-0,028$ \\
229 & 0,1201 & 0,1292 & $-0,009$ & 0,1870 & 0,2152 & $-0,028$ \\
325 & 0,1578 & 0,1052 & 0,052 & 0,1445 & 0,1395 & 0,005 \\
\hline
\end{tabular}

R. Bras. Eng. Agríc. Ambiental, v.14, n.3, p.279-287, 2010. 
piranômetros instalados na distância de dois metros sobre o dossel vegetal, no instante da passagem do sensor orbital; ainda na Tabela 5 estão os valores estimados pelo algoritmo SEBAL, para os pixeis correspondentes à localização das estações, com os erros absolutos em cada dia das imagens.

Observa que os valores estimados foram ligeiramente inferiores aos obtidos nos DJ 53, 101 e 325, em ambas as estações, enquanto os valores estimados com o SEBAL foram superiores nos DJ 213 e 229, nas duas estações.

Os erros encontrados na area de cana-de-açúcar estavam entre 0,005 e 0,045, enquanto na área de cerrado esses erros se situaram entre 0,006 a 0,031 ; verifica-se, portanto, que as diferenças encontradas aqui são bem inferiores às obtidas por Mendonça (2007), que encontrou variações nos valores estimados entre 0,05 e 0,16 em área cultivada com cana-deaçúcar, na região Norte Fluminense, trabalhando com imagens MODIS.

\section{CONCLUSÕES}

1. A metodologia proposta para as calibrações atmosféricas, foi bastante consistente para imagens Landsat TM neste estudo e para os produtos gerados a partir das mesmas.

2. O algoritmo SEBAL estimou satisfatoriamente os valores de albedo de superfícies sobre áreas de cerrado e de cana-de-açúcar, na região de Santa Rita do Passa Quatro, SP, consistentes com observações realizadas do albedo à superfície.

3. Foi possível determinar o albedo em toda a área de estudo com resultados muito consistentes com outros autores.

\section{LITERATURA CITADA}

Allen, R. G. Assessing integrity of weather data for use in reference evapotranspiration estimation. Journal of Irrigation and Drainage Engineering, v.122. n.2, p.97-106, 1996.

Allen, R. G.; Tasumi, M.; Trezza, R. SEBAL - Surface Energy Balance Algorithms for Land, Advanced training and users manual. Idaho: Implementation, v.1, p. 2002. 98p.

Allen, R. G.; Tasumi, M.; Trezza, R. Satellite-Based energy balance for mapping evapotraspiration with internalized calibration (METRIC) - Model. Journal of Irrigation and Drainage Engineering, v.133, n.4, p.380-394. 2007.

Bastiaanssen, W. G. M. Regionalization of surface flux densities and moisture indicators in composite terrain: A remote sensing approach under clear skies in Mediterranean climate. Netherlands: CIP Data Koninklijke Biblioteheek, Den Haag, 1995. 272p. PhD Tesis

Bastiaanssen, W. G. M. Sebal based sensible and latent heat fluxes in the irrigated Gediz Basin, Turkey. Journal of Hidrology, v.229, p.87-100, 2000.

Bastiaanssen, W. G. M.; Menenti, M.; Feddes, R. A.; Holtslag, A. A. M. A remote sensing surface energy balance algorithm for land (SEBAL) 1. Formulation. Journal of Hydrology, v.212-213, p.198-212, 1998.
Batalha, M. A. Análise da vegetação da ARIE Cerrado Pé-de-Gigante (Santa Rita do Passa Quatro, SP). Instituto de Biociências, São Paulo: USP, 1997. 184p. Dissertação Mestrado

Boegh, E.; Soegaard, H.; Thomsem, A. Evaluating evapotranspiration rates and surface conditions using Landsat TM to estimate atmospheric resistance and surface resistance. Remote Senssing of Enviromental, v.79, p.329-343, 2002.

Chandrapala, L.; Wimalasuriya, M. Satellite measurements supplemented with meteorological data to operationally estimate evaporation in Sri Lanka. Agricultural Water Management, v.58, p.89-107, 2003.

Garrison, J. D.; Adler, G. P. Estimation of precipitable water over the United State for aplication to the division of solar radiation into its direct and difuse coponents. Solar Energy, v.44, n.4, p.225-241, 1990.

Giongo, P. R.; Moura, G. B. A.; Silva, B. B.; Melo, J. S. P.; Medeiros, S. R. R. Albedo de superfície estimado por técnicas de sensoriamento remoto. In: Jornada de Ensino, Pesquisa e Extensão, 7, 2007, Recife, Anais... Recife: UFRPE, 2007, CD Rom

Hafeez M. M.; Chemim, Y.; Giesen, N. van de; Bouman B. A. M. Field evapotranspiration in central Luzon, Philippines, using different sensors: Landsat 7 ETM+, terra modis and aster. In: Simposium on Geospatial Theory, Processing And Applications, 2002, Ottawa, Anais... Ottawa: CIG, 2002, CD Rom

Iqbal, M. An introduction to solar radiation. New York: Academic Press. 1983. 212p.

Iziomons, M. G.; Mayer, H. On the variability and modeling of surface albedo and long-wave radiation components. Agricultural and Forest Meteoroly, v.111, p.141-152, 2002.

Liang, S. Narrowband to broadband conversations of land surface albedo _ I Algaritms. Remote Sensing of Environment, v.76, p.213-238, 2002.

Markham, B. L.; Barker, J. L. Thematic mapper bandpass solar exoatmospherical irradiances. International. Journal of Remote Sensing, v.8, n.3, p.517-523, 1987.

Mendonça, J. C. Estimativa da evapotranspiração regional utilizando imagens digitais orbitais na região Norte Fluminense. Rio de Janeiro: Universidade Estadual do Norte Fluminense Darcy Ribeiro, 2007. 145p. Tese Doutorado

Querino, C. A. S.; Moura, M. A. L.; Lyra, R. F. F.; Mariano, G. L. Avaliação e comparação de Radiação solar Global e albedo com ângulo zênital na região amazônica. Revista Brasileira de Meteorologia, v.21, n.3a, p.42-49, 2006.

Roerink, G. J. Bastiaanssen, W. G. M.; Chambouleyron, J.; Menenti, M. Relating crop water consumption to irrigation water supply to remote sensing. Water Resources Management, v.11, p.445-465. 1997.

Silva, B. B. da; Lopes, G. M.; Azevedo, P. V. Determinação do albedo de áreas irrigadas com base em imagens Landsat 5 TM. Revista Brasileira de Agrometeorologia, v.13, n.2, p.201-211. 2005a.

Silva, B. B. da, Lopes, G. M.; Azevedo, P. V. de. Balanço de radiação em áreas irrigadas utilizando imagens Landsat 5 TM. Revista Brasileira de Meteorologia, v.20, n.2. p.243-252, 2005b. 
SRTM. <http://srtm.csi.cgiar.org/SELECTION/listImages.asp> 18 Mai. 2007.

Timmermans, W. J.; Meijerink, A. M. J. Remotely sensed actual evapotranspiration, implications for ground water in Botswana, Journal of Applied Geohydrology, v.1, n.3/4, p.222-233, 1999.
Trezza, R. Evapotranspiration using a satellite-based surface energy balance with standardized ground control. Logan: Utah State University, 2002. 247p. Thesis Doctor

Varejão-Silva, M. A. Meteorologia e climatologia. 1.ed. Brasília: INMET, 2000. 532p. 